\title{
Modifications de la couleur du bois d'Abies grandis exposé à la lumière solaire
}

\author{
O Dirckx ${ }^{1}$, MC Triboulot-Trouy 2, A Merlin 2*, X Deglise 2 \\ 1 Société Dexter, 71700 Tournus; \\ 2 ESSTIB, université de Nancy I, laboratoire de photochimie appliquée, \\ BP 239, 54506 Vandceuvre-Lès-Nancy Cedex, France
}

(Reçu le 3 juin 1991; accepté le 10 juin 1992)

\begin{abstract}
Résumé - Le principal effet du rayonnement solaire sur le bois d'Abies grandis est une modification de sa couleur. On peut quantifier cette modification grâce au système CIE-LAB mais pour comprendre ce phénomène, une analyse de l'évolution des spectres d'absorption infrarouge et UV-visible est nécessaire. Les bandes d'absorption infrarouge ont pu être attribuées aux groupements chimiques présents en surface du bois. On note en cours d'irradiation, une diminution des groupements hydroxyles, une augmentation des groupements carbonyles et une désaromatisation. La lignine présente les mêmes évolutions de bandes que le bois d'Abies grandis. Ces évolutions sont sensibles à la présence d'oxygène. Les modifications dues au rayonnement ultraviolet sont plus importantes que celles produites par la lumière visible. L'observation des spectres UV-visible permet de suivre le jaunissement du bois d'Abies grandis. En début d'irradiation, la vitesse de jaunissement semble indépendante de l'atmosphère gazeuse. Le jaunissement est surtout imputable aux longueurs d'onde du domaine ultraviolet. Les modifications de couleur induites par l'exposition au rayonnement sont attribuées à la photodégradation de la lignine. L'absorption, directe ou indirecte, de l'énergie lumineuse par la lignine entrấnerait la formation d'un radical gaiacoxy coloré qui produirait des dérivés quinoniques colorés en présence d'oxygène ou des produits incolores en absence d'oxygène.
\end{abstract}

Abies grandis / lignine / couleur / spectroscopie / photodégradation

Summary - Wood photodiscolouration of Abies grandis under solar light exposure. In the present study, the effect of the solar-type irradiation was investigated by focusing mainly on fir (Abies grandis) discolouration. The CIE-LAB system was used for colour change measurements (table II, fig 3) and spectrometric methods such as infrared (IR) and ultraviolet visible (UV), were utilized to determine the mechanism. All the IR absorption bands were assigned to the different chemical groups of the wood surface (table $I V$ ). The absorption changes occurring during the solar-type exposure showed a decrease in the hydroxyl groups, an increase in the carbonyls and also desaromatization (table VII, fig 8). These changes were oxygen-dependent (fig 9). By cutting the UV or visible light emitted by the lamp with filters, it was possible to show that UV wavelengths were more important in the IR spectral modification (fig 10). Also, lignin photodegradation was similar in spectral evolution to fir degradation under solar-type irradiation. UV-visible spectroscopy was used to characterize the evolution of yellowing in fir, mainly due to the UV wavelengths (fig 13). At the beginning of light exposure, the yellowing rate seemed to be independent of the gaseous atmosphere. Lignin seemed to be responsible for the light absorption of wood and for discolouration (fig 15), and the main source of radicals. These gaiacoxy radicals appear to form coloured quinonoid structures with oxygen, and colourless products without it (fig 19).

Abies grandis / lignin / photodiscolouration / FTIR absorption spectrocopy / UV and visible spectroscopy

* Correspondance et tirés à part 


\section{INTRODUCTION}

La compréhension du comportement photochimique du bois soumis à une irradiation de type solaire est apparue nécessaire à la suite de travaux effectués sur les systèmes bois-finitions transparentes (Gaillard, 1984) qui avaient montré que, en présence d'un rayonnement ultravioletvisible, non seulement la résine de finition mais aussi le bois se dégradaient.

Le but de cette étude à caractère fondamental est d'analyser les modifications moléculaires qui s'opèrent au sein de la structure du bois afin d'établir des mécanismes qui permettent d'envisager les remèdes mieux adaptés à sa protection à la lumière.

La plupart des travaux à caractère fondamental sur les composés lignocellulosiques ont été menés sur des molécules modèles (Lin et Kringstadt, 1970; Gellerstedt et Pettersson, 1975, 1977, 1980; Castellan et al, 1985; Vanucci et al, 1988; Castellan et al, 1989a,b) ou sur les constituants principaux du bois (lignine, cellulose, hémicellulose) à l'état isolé (Lin et Kringstadt, 1971; Gierer et Lin, 1972; Hon, 1975; Merlin et Fouassier, 1980; Le Nest et al, 1982; Nagaty et al, 1982; Schmitt, 1984; Neumann et al, 1986a,b; Castellan et al, 1990).

Afin d'éviter d'une part les difficultés d'extrapolation au matériau lui-même et pour tenir compte d'autre part des liaisons chimiques et des transferts d'énergie possibles au sein du matériau entre les différents constituants, nous avons choisi de travailler directement sur le bois (Dirckx, 1988; Mazet, 1988) et non sur les modèles.

\section{MATÉRIELS ET MÉTHODES}

\section{Essence utilisée}

Nous avons essentiellement travaillé sur des échantillons de bois de sapin de Vancouver
(Abies grandis $\mathrm{L}$ ). Cette essence a été choisie d'une part pour la simplicité de son plan ligneux, son aspect relativement homogène, sa grande largeur de cernes et d'autre part pour la quasiabsence de substances extractibles colorées. Ainsi, le comportement à la lumière du bois d'Abies grandis sera représentatif de celui des constituants principaux communs à toutes les essences et ne sera influencé d'aucune manière par des éléments spécifiques à une essence particulière.

\section{Préparation des échantillons}

Deux types d'échantillons ont été réalisés en fonction de la technique d'analyse spectroscopique qui leur est appliquée pour suivre la photodégradation.

\section{Coupes minces (épaisseur variant de $50-80 \mu \mathrm{m}$ )}

Elles ont été préparées sur un microtome Reichert type OME dans le plan LT (longitudinaltangentiel) où le bois est le plus homogène. Ces coupes ont été réalisées préférentiellement dans le bois initial dans des zones où le rayon de courbure des cernes annuels est grand.

\section{Échantillons massifs (épaisseur variant de 3-5 $\mathrm{mm}$ ) dont la face $L T$ a été aplanie au microtome jusqu'au bois de printemps}

Le taux moyen d'humidité de ces échantillons était de $10 \%$. En raison de leur faible épaisseur, l'équilibre hygroscopique avec le milieu est atteint rapidement.

\section{Constituants principaux du bois}

Les lignines du bois d'Abies grandis ont été extraites par acidolyse dans le dioxanne. Cette extraction de rendement très faible (1-2\% par rapport au bois sec) permet d'obtenir des lignines peu dégradées. La cellulose a été extraite du bois de peuplier (Populus canenscens $\mathrm{Sm}$ ). Les échantillons d'hémicellulose proviennent de bois 
de séquoïa (Sequoïa sempervirens) et de roseau (Arundo donax), leur analyse détaillée est donnée sur le tableau I.

\section{Dispositif d'irradiation}

Les modifications naturelles de la couleur du bois ont le plus souvent pour origine la lumière du jour. Pour des raisons pratiques (standardisation des irradiations et accélération de la photodégradation), nous avons simulé le rayonnement solaire à l'aide d'une lampe à vapeur de mercure haute pression du type Hanau TQ 150. Le flux lumineux émis par cette source à la distance utilisée pour l'exposition des échantillons a étè mesuré à $360 \mathrm{~nm}$ à l'aide d'un radiomère. Notons qu'à cette longueur d'onde, le flux émis par le soleil à la surface de la terre est d'environ $0,1 \mathrm{~mW} / \mathrm{cm}^{2}$ (Lablache-Combier, 1985). En plaçant un thermocouple sur la surface d'un échantillon exposé au rayonnement, nous avons mesuré une élévation de température de $5-6^{\circ} \mathrm{C}$ en régime permanent et pour les flux lumineux les plus intenses. Nous avons vérifié que cet échauffement est sans effet sur les phénomènes de vieillissement observés lors de l'irradiation lumineuse (DirckX, 1984).

Pour séparer les effets du rayonnement ultraviolet et ceux de la lumière visible, nous avons utilisé un filtre passe-bande centré à $335 \mathrm{~nm}$ et un filtre passe-haut coupant à $400 \mathrm{~nm}$.

Tableau I. Analyse chimique des hémicelluloses provenant de bois de sequoīa et de Roseau.

Hémicellulose Résultat
analysée

Xylane de roseau $82 \%$ de xylose

(Arundo donax) $7 \%$ d'arabinose $10 \%$ d'acide glucuronique

Glucomannane purifiée à partir de Séquoïa (Sequoïa smpervirens)

$35 \%$ de glucose

$60 \%$ de mannose

traces de galactose et xylose

\section{Analyse spectroscopique}

Suivant la nature des échantillons, nous avons mis en œuvre 2 techniques d'analyse spectrocopique : absorption infrarouge et ultravioletvisible pour les coupes minces, réflexion pour les échantillons massifs. Les mesures spectrophotométriques d'absorption des corps solides, bien que nécessitant des échantillons très minces, permettent de garder intacte la structure des matériaux et de l'observer dans son entière cohésion.

\section{Spectrocopie d'absorption infrarouge}

Nous avons suivi l'absorption infrarouge des coupes de bois grâce à un spectromètre à transformée de Fourier (FTIR1750 - Perkin Elmer) qui permet des analyses quantitatives correctes jusqu'à une densité optique de 3 .

\section{Spectroscopie ultraviolet-visible}

Pour chacun des modes d'utilisation (réflexion et absorption), nous avons adjoint à un spectrophotomètre Perkin Elmer Lambda 3 une sphère d'intégration qui permet de collecter la lumière dispersée dans toutes les directions pour des échantillons diffusant la lumière. Cette sphère d'intégration permet également de mesurer la couleur des échantillons par le calcul des valeurs tristimulaires $X Y$ et $Z$ sous l'illuminant $D_{65}$ avec un angle d'ouverture de $2^{\circ}$.

Ces spectromètres ultraviolet-visible et FTIR sont couplés à des stations de données qui facilitent l'analyse et le traitement des spectres. Elles permettent notamment de construire les courbes de différence de 2 spectres pour s'affranchir des écarts de ligne de base. Ainsi les spectres d'absorption infrarouge ont été ramenés à une valeur identique de la densité optique à $1800 \mathrm{~cm}^{-1}$, longueur d'onde où aucun des constituants n'absorbe. Aucune normation n'a été nécessaire pour les spectres d'absorption et de réflexion ultraviolet-visible.

Pour tenir compte de la variabilité de la couleur des échantillons liée à leur nature et à leur état de surface, nous avons pris en compte les modifications de l'absorption UV-visible et infrarouge au cours d'une même irradiation de cinq échantillons différents. 


\section{Cellules d'analyse}

Pour apprécier le rôle de l'oxygène dans les processus de photocoloration du bois, nous avons conçu des cellules permettant l'irradiation et l'enregistrement des spectres d'absorption infrarouge et ultraviolet-visible dans différentes atmosphères gazeuses (fig $1 \mathrm{a}, \mathrm{b}$ ).

\section{Cellule pour le suivi par spectroscopie d'absorption infrarouge (fig 1a)}

Elle est constituée d'un tube de pyrex fermé à ses 2 extrémités par des fenêtres en chlorure

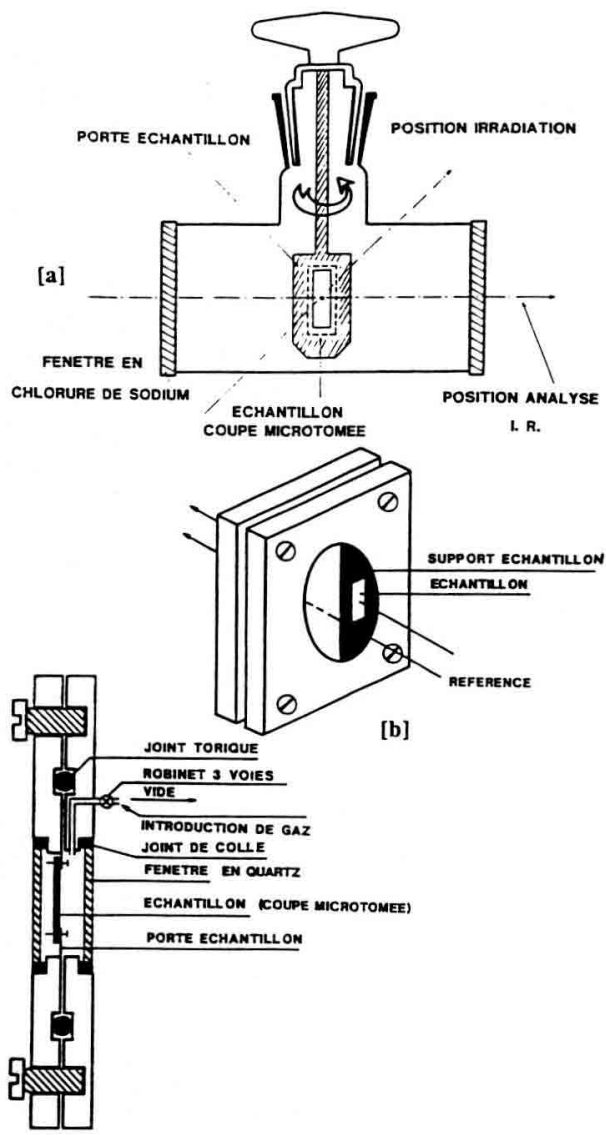

Fig 1.a. Cellule pour l'analyse en spectroscopie d'absorption infrarouge. b. Cellule pour l'analyse en spectroscopie d'absorption UV-Visible. de sodium, matériau transparent au rayonnement infrarouge. Le porte-échantillon est solidaire d'un rodage qui permet 2 orientations : l'une correspond à l'irradiation à travers le pyrex; l'autre, obtenue par rotation de $90^{\circ}$ (échantillon perpendiculaire à l'axe de la cellule), permet l'analyse infrarouge à travers les fenêtres en chlorure de sodium.

\section{Cellule pour le suivi par spectroscopie d'absorption ultraviolet-visible (fig 1b)}

Le faisceau témoin traverse uniquement les 2 plaques de quartz (matériau transparent au rayonnement ultraviolet-visible) tandis que le faisceau d'analyse traverse la coupe qui a été irradiée.

Ces 2 cellules peuvent être raccordées à une station de vide qui permet d'obtenir des pressions de l'ordre de $10^{-4} \mathrm{~mm}$ de mercure. $\dot{A}$ l'aide d'un robinet à trois voies, on peut alors introduire dans la cellule les différentes atmosphères gazeuses.

\section{RÉSULTATS}

La détérioration du bois par le rayonnement solaire se traduit essentiellement par des modifications de sa couleur initiale. $\mathrm{Ce}$ paramètre peut donc être utilisé pour suivre la photodégradation des échantillons. Pour quantifier la couleur, les coloristes ont mis au point différents systèmes à partir de la mesure des valeurs tristimulaires (Chrisment, 1988; Kovaliski, 1990). Le plus utilisé est le système CIE Lab (Decarreau, 1988; Dordet, 1990). Dans cet espace de représentation des couleurs, un point est repéré par la luminance qui varie de 0 (noir) à 100 (blanc de référence) et par les coordonnées $a$ et $b$ qui correspondent respectivement aux 2 couples de couleurs complémentaires rouge-vert et bleujaune. Ce système est bien adapté à la mesure de la couleur du bois (Nemeth, 1982; Janin, 1986; Flot, 1988).

Sur le tableau II, nous avons reporté les valeurs moyennes des coordonnées $L, a, b$ obtenues sur des échantillons massifs 
Tableau II. Quantification de la couleur de différentes essences dans le système $\mathrm{CIE} L A B$ : valeurs moyennes des coordonnées chromatiques L, $a$ et $b$.

\begin{tabular}{ccccc}
\hline & & & \\
$\begin{array}{c}\text { Sapin de } \\
\text { Vancouver } \\
\text { Abies } \\
\text { grandis }\end{array}$ & $\begin{array}{c}\text { Hêtre } \\
\text { Fagus } \\
\text { silvatica }\end{array}$ & $\begin{array}{c}\text { Noyer } \\
\text { Juglans Sp }\end{array}$ & Amarante \\
\hline L & 80 & 71 & 56 & 42 \\
a & $-0,4$ & 4,3 & 2,8 & 6,5 \\
b & 19 & 20,8 & 14,5 & 5 \\
\hline
\end{tabular}

(30 x $20 \times 5 \mathrm{~mm}$ ) de différentes essences. Pour chaque échantillon, les coordonnées chromatiques ont été mesurées en 10 points régulièrement espacés. Ces mesures sont évidemment sensibles à l'état de surface de l'échantillon et à la nature de la section mesurée (tangentielle, radiale, transversable) (Hofmann, 1987) et n'ont pas la prétention d'être caractéristiques des essences considérées.

Nous pouvons noter :

- la forte luminance du bois d'Abies grandis par rapport aux bois colorés;

- la dominance jaune de la couleur des bois de sapin de Vancouver (Abies grandis), de hêtre (Fagus silvatica) et de noyer (Juglans sp);

- les valeurs voisines des composantes a et $b$ pour le bois d'Amarante.

La construction de cet espace CIE LAB de représentation des couleurs est telle que la mesure des écarts de couleur $\Delta E$ entre 2 points est donnée par la distance cartésienne les séparant :

$$
\Delta E=\left(\Delta L^{2}+\Delta a^{2}+\Delta b^{2}\right)^{1 / 2}
$$

$\Delta L, \Delta a$ et $\Delta b$ sont les différentes respectives en luminance et coordonnées chromatiques entre 2 points de couleur.
Les modifications de couleur induites par l'exposition d'un échantillon de bois à un rayonnement de type solaire peuvent être quantifiées par ces paramètres $\Delta L$ et $\Delta E$. Au cours de l'irradiation d'un échantillon de bois d'Abies grandis, nous notons une augmentation importante de l'écart de couleur $\Delta E$ qui est due essentiellement à la variation de luminance $\Delta L$ (fig 2). Les changements de tonalité exprimés dans le plan (a,b) (fig 3) montrent que l'échantillon jaunit tout en rougissant, $\Delta a$ et $\Delta b$ augmentent simultanément. Ainsi, après $500 \mathrm{~h}$ d'exposition avec un flux photonique moyen de 2 $\mathrm{mW} / \mathrm{cm}^{2}$ à $360 \mathrm{~nm}$, nous obtenon un écart de couleur $\Delta E$ de $15(\Delta a \approx 4,6$ et $\Delta b \approx 5,4)$. Cette variation de couleur est visible à l'œil, l'échantillon devenant ocre foncé. Nous rap-
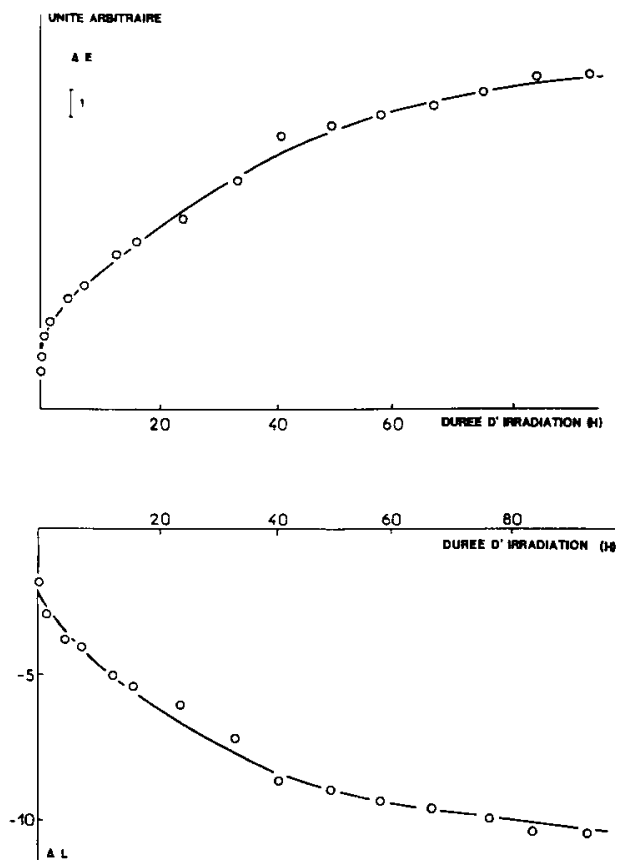

Fig 2. Évolution de l'écart global de couleur $\Delta E$ et de la luminance $\Delta L$ du bois d'Abies grandis en fonction du temps d'irradiation. 


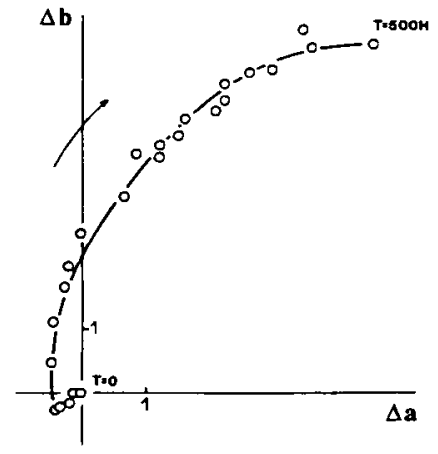

Fig 3. Évolution des coordonnées chromatiques de bois d'Abies grandis en fonction du temps d'irradiation.

pelons que l'œil est sensible à une variation de luminance de $3 \%$ (Mc Ginnes et Rosen, 1984). Minemura et Umehara (1979) ont établi une correspondance entre les valeurs des écarts de couleurs $\Delta E$ et l'estimation visuelle (tableau III).

La sensation de couleur est produite par la lumière transmise ou réfléchie par un objet uniquement dans le domaine spectral du visible (400-800 nm). Ces mesures dans le système CIE LAB représentent la modification globale de l'aspect coloré des échantillons par intégration sur le

Tableau III. Correspondance entre l'écart de couleur $\Delta \mathrm{E}$ et l'appréciation visuelle entre deux points dans le système CIE LAB.

\begin{tabular}{lc} 
Appréciation visuelle & $\Delta E$ \\
\hline Trace & \\
Légère & $0-0,5$ \\
Notable & $0,5-1,5$ \\
Appréciable & $1,5-3$ \\
Importante & $3-6$ \\
Très importante & $6-12$ \\
& $>12$ \\
\hline
\end{tabular}

domaine de sensibilité de l'œil. Pour proposer une interprétation moléculaire des phénomènes, il est nécessaire de suivre les modifications d'absorption (ou de réfléxion) dans une zone spectrale plus étendue allant de l'ultraviolet à l'infrarouge.

\section{Suivi de la photodégradation du bois d'Abies grandis par spectroscopie infrarouge}

La spectroscopie d'absorption infrarouge permet de connaître la nature des différents groupements chimiques présents dans le bois et donc de suivre les modifications de ces fonctions chimiques engendrées par l'exposition des échantillons au rayonnement solaire. II est souvent difficile d'interpréter de façon précise les bandes d'absorption infrarouge car leur intensité et leur localisation peuvent varier de façon significative selon l'environnement et l'abondance des groupements chimiques. Pour cette raison, nous avons étudié, en plus du sapin de Vancouver (Abies grandis), 2 essences feuillues, le hêtre (Fagus silvatica) et le noyer (Juglans $\mathrm{sp}$ ), qui se différencient par leur teneur en constituants principaux (tableau IV) (Fengel et Wegener, 1984).

L'analyse des spectres d'absorption infrarouge de ces différentes essences et des principaux constituants du bois a permis, en s'aidant des résultats obtenus sur d'autres essences (Marchessault, 1962; Harrington et al, 1964; Sarkanen et al, 1967; Liang et al, 1968; Michell et al, 1969; Chow, 1971; Nagaty et al, 1982; Pecina, 1982), d'identifier la totalité des bandes d'absorption présentes dans un spectre infrarouge du bois (tableau $V$ ) et de préciser le ou les constituants concernés (tableau VI). Les différences les plus marquées entre les spectres d'absorption infrarouge des essences feuillues et résineuses sont observées dans la zone spectrale 850$1270 \mathrm{~cm}^{-1}$ (fig 4). 
Tableau IV. Teneurs moyennes en constituants principaux de différentes essences (par rapport au poids anhydre).

\begin{tabular}{lcccc}
\hline Essence & Cellulose (\%) & Lignine (\%) & \multicolumn{2}{c}{ Hémicellulose (\%) } \\
\cline { 4 - 5 } & & & Pentosane & Hexosane \\
\hline $\begin{array}{l}\text { Sapin de Vancouver } \\
\text { (Abies grandis) }\end{array}$ & 42 & 29 & 11 & 13 \\
$\begin{array}{l}\text { Hêtre } \\
\text { (Fagus silvatica) }\end{array}$ & 44 & 22 & 20 & 10 \\
$\begin{array}{l}\text { Noyer } \\
\text { (Juglans sp) }\end{array}$ & 40 & 29 & 12 & \\
\hline
\end{tabular}

Tableau V. Comparaison des spectres d'absorption infrarouge de différentes essences : localisation des bandes des bois d'Abies grandis, de Fagus silvatica et de Juglans sp (nombre d'onde en $\mathrm{cm}^{-1}$ ).

\begin{tabular}{ccc}
\hline $\begin{array}{c}\text { Abies } \\
\text { grandis } \\
\text { (Sapin de Vancouver) }\end{array}$ & $\begin{array}{c}\text { Fagus } \\
\text { silvatica } \\
\text { (Hêtre) }\end{array}$ & $\begin{array}{c}\text { Juglans sp } \\
\text { (Noyer) }\end{array}$ \\
\hline 3355 & 3338 & 3365 \\
$2933^{\mathrm{e}}$ & $2937^{\mathrm{e}}$ & $2942^{\mathrm{e}}$ \\
2900 & 2901 & 2906 \\
1734 & 1735 & 1733 \\
$1650^{\mathrm{L}}$ & $1650^{\mathrm{L}}$ & $1650^{\mathrm{L}}$ \\
1605 & 1593 & 1596 \\
1510 & 1505 & 1505 \\
1455 & 1460 & 1461 \\
1425 & 1422 & 1425 \\
1371 & 1371 & 1371 \\
$1335^{\mathrm{e}}$ & 1332 & \\
1318 & 1322 & 1322 \\
1266 & 1235 & $1235^{\mathrm{L}}$ \\
$1230^{\circ}$ & 1351 \\
$1200^{\circ}$ & 1160 & 1161 \\
1163 & 1108 & 1109 \\
1110 & 1054 & 1054 \\
1060 & 1034 & 1035 \\
1036 & 899 & 899 \\
898 & & \\
$863^{\mathrm{e}}$ & & \\
806 & & \\
\hline
\end{tabular}

e : épaulement; $L$ : bande large.

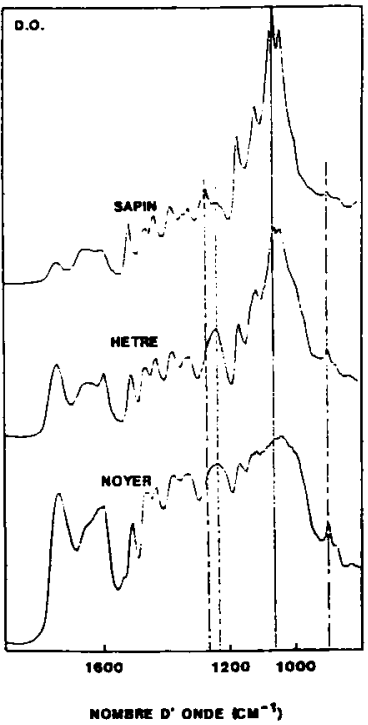

Fig 4. Spectres d'absorption infrarouge des différentes essences de bois.

Les bandes à $1265 \mathrm{~cm}^{-1}$ et $1235 \mathrm{~cm}^{-1}$ sont attribuées aux élongations de la liaison $\mathrm{C}-\mathrm{O}$ des groupes méthoxyles $\left(-\mathrm{OCH}_{3}\right)$ respectivement des unités gaïacyles et syringyles. Dans le cas du bois de sapin de 
Tableau VI. Localisation et attribution des bandes d'absorption infrarouge du bois d'Abies grandis et des principaux constituants du bois (nombre d'onde en $\mathrm{cm}^{-1}$ ).

\begin{tabular}{|c|c|c|c|c|c|}
\hline $\begin{array}{l}\text { Abies } \\
\text { grandis }\end{array}$ & Cellulose & Lignine & $\begin{array}{c}\text { Hémic } \\
\text { Xylane }\end{array}$ & $\begin{array}{l}\text { Muloses } \\
\text { Mannane }\end{array}$ & $\begin{array}{l}\text { Attribution des bandes } \\
\text { d'absorption }\end{array}$ \\
\hline $\begin{array}{l}3355 \\
2933^{\mathrm{e}} \\
2900\end{array}$ & $\begin{array}{l}3347 \\
2900\end{array}$ & $\begin{array}{l}3392 \\
2924 \\
2850\end{array}$ & $\begin{array}{l}3402 \\
2921 \\
2899 \mathrm{e}\end{array}$ & $\begin{array}{l}3402 \\
2923 \\
2885^{\mathrm{e}}\end{array}$ & $\begin{array}{l}v(\mathrm{O}-\mathrm{H}) \\
v_{\text {as }}\left(\mathrm{CH}_{2}\right) \\
v(\mathrm{C}-\mathrm{H}) \text { [aliphatique] } \\
v\left(\mathrm{CH}_{2}\right) \text { [groupes méthoxy aromatiques] } \\
\text { (non visible dans le spectre du bois) }\end{array}$ \\
\hline $\begin{array}{l}1734 \\
1650^{\mathrm{L}}\end{array}$ & 1640 & $\begin{array}{l}1720^{\mathrm{e}} \\
1670^{\mathrm{e}}\end{array}$ & $1630^{L}$ & $1640^{L}$ & $\begin{array}{l}\vee(\mathrm{C}=\mathrm{O}) \\
1680 \vee(\mathrm{C}=0) \\
\text { massif } \\
1638 \delta(\mathrm{H}-\mathrm{O}-\mathrm{H})\end{array}$ \\
\hline $\begin{array}{l}1605 \\
1510 \\
1455 \\
1425\end{array}$ & $\begin{array}{l}1455^{\circ} \\
1425\end{array}$ & $\begin{array}{l}1598 \\
1506 \\
1458 \\
1419\end{array}$ & $\begin{array}{l}1463^{e} \\
1408\end{array}$ & $1417^{e}$ & $\begin{array}{l}\delta(\mathrm{C}=\mathrm{C}) \text { [cycles aromatiques] } \\
\delta(\mathrm{C}=\mathrm{C}) \text { [cycles aromatiques] } \\
\delta\left(\mathrm{CH}_{2}\right) \text { et }\left(\mathrm{CH}_{3}\right) \\
\delta \text { cisaillement }\left[\left(\mathrm{CH}_{2}\right) \text { et }\left(\mathrm{CH}_{3}\right)\right. \\
\text { des lignines })\end{array}$ \\
\hline 1371 & 1368 & $\begin{array}{l}1385^{\mathrm{e}} \\
1370^{\circ}\end{array}$ & 1386 & & $\begin{array}{l}\delta(\mathrm{CH}) \\
\delta \text { sy }\left(\mathrm{CH}_{3}\right)\end{array}$ \\
\hline $1335^{e}$ & 1333 & $\begin{array}{l}1352 \\
1330^{e}\end{array}$ & & & $\gamma(\mathrm{CH} 2)$ et $\delta(\mathrm{O}-\mathrm{H})$ \\
\hline $\begin{array}{l}1318 \\
1266 \\
1230^{e} \\
1200^{e}\end{array}$ & $\begin{array}{l}1314 \\
1280 \\
1230^{e} \\
1200\end{array}$ & $\begin{array}{l}1266 \\
1222\end{array}$ & $\begin{array}{l}1313^{e} \\
1252 \\
1208^{e}\end{array}$ & $\begin{array}{l}1309 \\
1260 \\
1208 \mathrm{e}\end{array}$ & $\begin{array}{l}\delta\left(\mathrm{CH}_{2}\right) \text { et } \delta(\mathrm{O}-\mathrm{H}) \\
v(\mathrm{C}-\mathrm{O}) \text { [lignines et mannanes] } \\
v(\mathrm{C}-\mathrm{O}) \text { [lignines] } \\
d\left(\mathrm{CH}_{2}\right) \text { et } \mathrm{d}(\mathrm{O}-\mathrm{H}) \\
v_{\text {as }}(\mathrm{C}-\mathrm{O}-\mathrm{C})\end{array}$ \\
\hline $\begin{array}{r}1163 \\
1110 \\
1060 \\
1036 \\
898\end{array}$ & $\begin{array}{r}1160 \\
1109 \\
1058 \\
1032 \\
895\end{array}$ & $\begin{array}{l}1134 \\
1080 \\
1032\end{array}$ & $\begin{array}{l}1165 \\
1115^{e} \\
1082^{e} \\
1045 \\
898\end{array}$ & $\begin{array}{l}1152 \\
1092 \\
1062 \\
1030 \\
895\end{array}$ & $\begin{array}{l}v_{\text {as }}(C-O-C) \\
v(C-O) \text { et } v(O-H) \\
v(C-O) \\
v_{\text {sy }}(C-O-C) \\
v(C-C) \text { [cycles glucosidiques } \\
\text { dans les liaisons } \beta \\
\text { des groupements } \\
\text { polysaccharides }\end{array}$ \\
\hline $863^{e}$ & & 853 & & 872 & $\begin{array}{l}\delta(\mathrm{C}-\mathrm{H}) \\
v[\text { mannanes }]\end{array}$ \\
\hline 806 & & 812 & & 813 & $\begin{array}{l}\delta(\mathrm{C}-\mathrm{C}) \\
v[\text { mannanes] }\end{array}$ \\
\hline
\end{tabular}

e : épaulement; $L$ : large bande; $v$ : vibration d'élongation ou de valence; $\delta$ : vibration de déformation; $\gamma$ : vibration de balancement. 
Vancouver, qui contient de la lignine riche en unités gaïacyles (Sarkanen et al, 1967), la bande à $1265 \mathrm{~cm}^{-1}$ est nettement plus intense que la bande à $1235 \mathrm{~cm}^{-1}$. Ces 2 bandes ont des intensités voisines dans les spectres des bois de hêtre et de noyer correspondant à des teneurs équivalentes en unités gaïacyles et syringyles. Les quantités différentes des mannanes et des xylanes dans les gymnospermes (résineux) et angiospermes (feuillus) peuvent également contribuer aux différences d'intensité entre ces 2 bandes d'absorption à $1265 \mathrm{~cm}^{-1}$ et $1235 \mathrm{~cm}^{-1}$ (Michell et al, 1969).

Cette différence entre les feuillus riches en xylane et les résineux riches en mannanes (Harrington et al, 1964) se retrouve dans d'autres régions du spectre infrarouge :

$-1060 \mathrm{~cm}^{-1}$ : absorption plus intense dans le cas du sapin de Vancouver (mannes prépondérants);

$-898 \mathrm{~cm}^{-1}$ : absorption plus intense dans le cas des feuillus (xylanes prépondérants);

- les bandes à $863 \mathrm{~cm}^{-1}$ et $806 \mathrm{~cm}^{-1}$ spécifiques des mannanes sont uniquement visibles dans le spectre de l'Abies grandis.

Après une longue durée d'irradiation, les spectres d'absorption infrarouge des 3 essences présentent des modifications voisines. Les bandes d'absorption les plus affectées par l'exposition au rayonnement ont pu être attribuées :

- $3355 \mathrm{~cm}^{-1}$ : cette absorption due aux groupements hydroxyles diminue fortement;

- $1735 \mathrm{~cm}^{-1}$ : cette bande attribuée à la vibration d'élongation des groupements carbonyles est celle dont l'augmentation est la plus spectaculaire (fig 5);

- zone spectrale $1680-1580 \mathrm{~cm}^{-1}$ : elle est difficilement interprétable car l'absorption est la résultante des vibrations de défor-

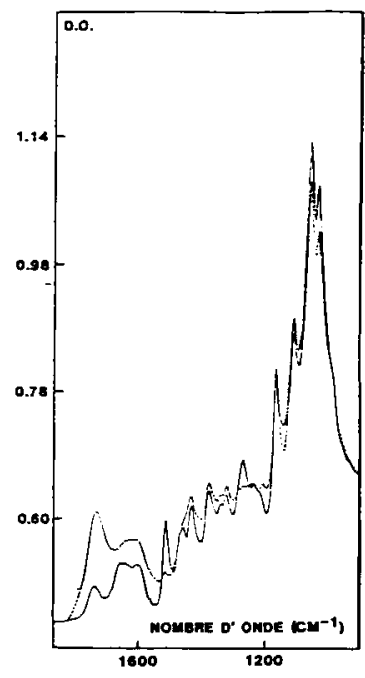

Fig 5. Spectres d'absorption infrarouge de bois d'Abies grandis - avant irradiation; -.- après $1690 \mathrm{~h}$ d'irradiation.

mation de la molécule d'eau $\left(1638 \mathrm{~cm}^{-1}\right)$, des vibrations d'élongation $\mathrm{C}=0$ des cétones conjuguées $\left(1660 \mathrm{~cm}^{-1}\right)$ et des vibrations d'élongation $\mathrm{C}=\mathrm{C}$ des noyaux aromatiques (1 $\left.600 \mathrm{~cm}^{-1}\right)$;

- la chute de l'absorption à $1510 \mathrm{~cm}^{-1}$ (vibration de déformation $\mathrm{C}=\mathrm{C}$ dans les cycles aromatiques) observée pour les 3 essences, montre la désaromatisation de la structure du matériau au cours de l'irradiation. Dans le cas du sapin de Vancouver (fig 11b), cette bande a pratiquement disparu après une longue irradiation;

- les autres bandes $(1160,1110,1060$ et $1035 \mathrm{~cm}^{-1}$ ) attribuées aux élongations $\mathrm{C}$ O subissent de même une diminution d'intensité.

Les différences des modifications des spectres d'absorption infrarouge des 3 essences sont peu marquées. L'absence de singularité dans le cas du sapin de Van- 
couver conforte notre choix de cette essence comme modèle du comportement photochimique du bois.

La même étude spectroscopique des 2 principaux constituants du bois, la lignine (fig 6a) et la cellulose (fig 6b) montre que la cellulose isolée résiste bien à ce type d'irradiation, son spectre subissant peu de modifications. Par contre, les évolutions des bandes d'absorption infrarouge de la lignine sont très proches de celles observées pour le bois d'Abies grandis, en particulier dans la zone spectrale 1800-1500 $\mathrm{cm}^{-1}$ (fig 6a) :

[a]

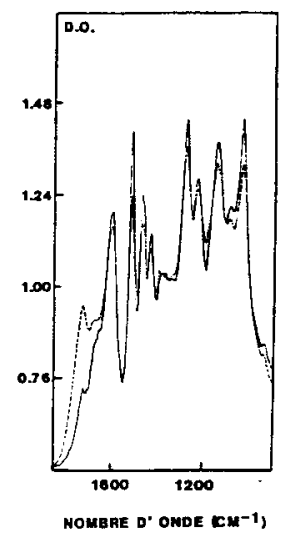

[b]

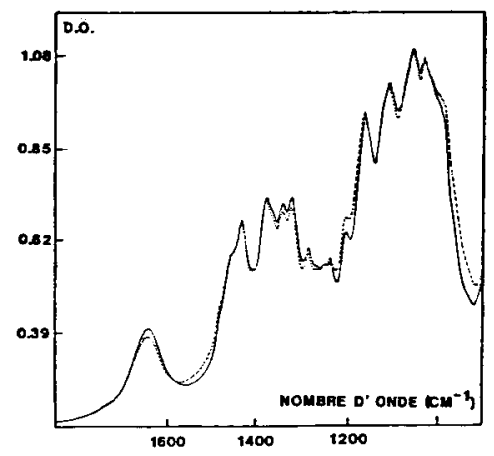

Fig 6. Spectres d'absorption infrarouge de la lignine (a) et de la cellulose (b); - avec d'irradiation; -.- après 400 heures d'irradiation.
$-1730 \mathrm{~cm}^{-1}$ : la faible absorption initiale due aux vibrations d'élongation $\mathrm{C}=\mathrm{O}$ des groupements carbonyles voit son intensité augmenter de façon importante;

$-1670 \mathrm{~cm}^{-1}$ : l'épaulement traduisant les vibrations d'élongation des groupements $\mathrm{C}=0$ des cétones aromatiques conjuguées semble subir une augmentation d'intensité;

$-1598 \mathrm{~cm}^{-1}$ et $1506 \mathrm{~cm}^{-1}$ : ces 2 bandes d'absorption attribuées aux vibrations de déformation $\mathrm{C}=\mathrm{C}$ dans les cycles aromatiques voient leur intensité diminuer.

Ces observations montrent qu'à l'intérieur du bois, ce sont les lignines qui subissent le plus de transformations et qu'il y a formation de composés carbonylés (1 735 $\mathrm{cm}^{-1}$ et $1660 \mathrm{~cm}^{-1}$ ) dont certains doivent participer aux modifications de la couleur du matériau. L'évolution des 3 bandes d'absorption infrarouge pour lesquelles les modifications sont les plus marquées 1735,1660 et $1510 \mathrm{~cm}^{-1}$ peut être suivie en représentant la différence de densité optique entre les coupes de bois d'Abies grandis irradiée et non irradiée (fig 7 a,b,c). Pour ces 3 nombres d'onde, il apparaît qu'au-delà de $1500 \mathrm{~h}$, les évolutions des bandes se stabilisent et tendent vers un état stationnaire. Durant les 100 premières $\mathrm{h}$ d'irradiation, les absorptions à $1735 \mathrm{~cm}^{-1}$ (vibration d'élongation $\mathrm{C}=\mathrm{O}$ dans les composés carbonylés) et à 1510 $\mathrm{cm}^{-1}$ (vibration d'élongation $\mathrm{C}=\mathrm{C}$ des noyaux aromatiques) varient rapidement. Pour la bande à $1735 \mathrm{~cm}^{-1}$, l'état stationnaire correspond à une différence de densité opatique plus importante que pour la bande à $1510 \mathrm{~cm}^{-1}$ (tableau VII). La variation de l'absorption à $1660 \mathrm{~cm}^{-1}$ (vibration d'élongation $\mathrm{C}=\mathrm{O}$ dans les cétones conjuguées (fig 9c) semble résulter d'une compétition entre plusieurs bandes d'absorption évoluant de façon opposée : en début d'irradiation, l'absorption diminue puis, après quelques $h$, son intensité se met à augmenter. À l'état stationnaire, l'augmen- 
[a]
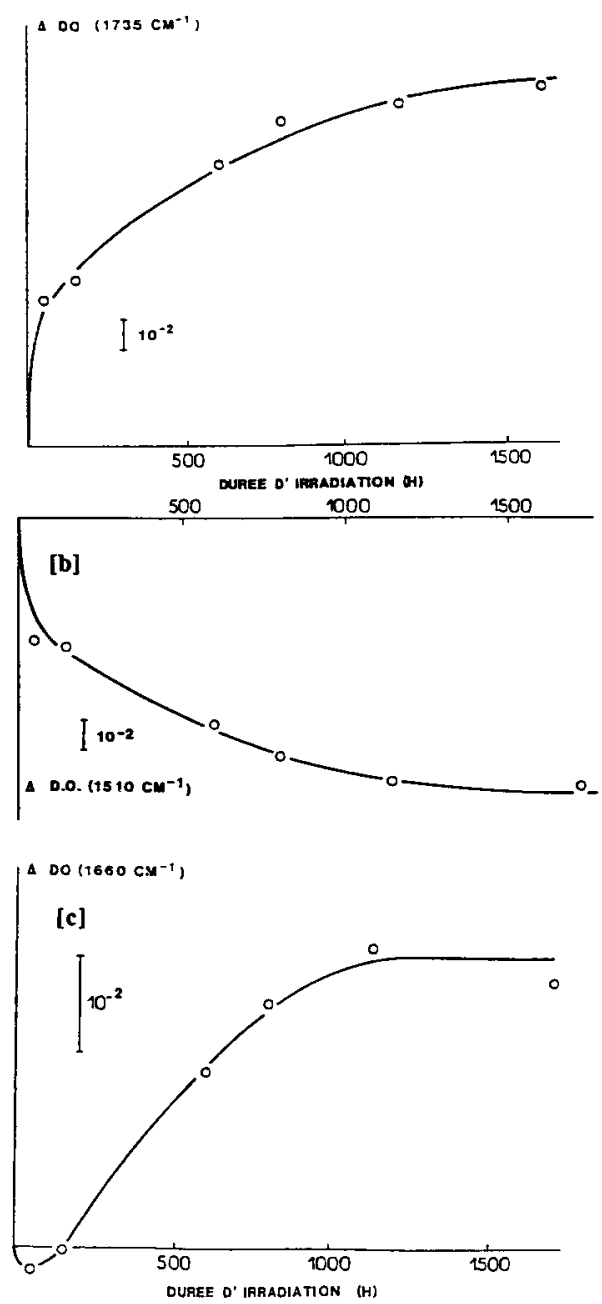

Fig 7. Évolution de la densité optique à 1735 $\mathrm{cm}^{-1}$ (a), à $1510 \mathrm{~cm}^{-1}$ (b) et à $1660 \mathrm{~cm}^{-1}$ (c) en fonction du temps d'irradiation.

tation de la densité optique de l'échantillon irradié reste faible (tableau VII).

L'évolution des spectres d'absorption infrarouge du bois d'Abies grandis dans la zone $1800-1500 \mathrm{~cm}^{-1}$ est sensible à la présence d'oxygène. Cette étude a été ré-
Tableau VII.Augmentation de la densité optique $D O$ à l'état stationnaire des bandes d'absorption infrarouge à 1735,1660 et $1510 \mathrm{~cm}^{-1}$ d'un échantillon de bois d'Abies grandis irradié; $\triangle D O$ $=(D O$ à l'état stationnaire $-D O$ avant irradiation).

\begin{tabular}{lr}
\hline$v\left(\mathrm{~cm}^{-1}\right)$ & $\Delta D O$ \\
\hline 1735 & $13.10^{-2}$ \\
1660 & $3.10^{-2}$ \\
1510 & $-9.10^{-2}$ \\
\hline
\end{tabular}

alisée en comparant les spectres d'absorption infrarouge d'une coupe irradiée en atmosphère d'azote ou d'oxygène que l'on introduit après avoir fait le vide dans la celluie d'analyse. L'absorption à $1735 \mathrm{~cm}^{-1}$ (fig 10a) croît 2 fois plus vite dans l'oxygène que dans l'air (tableau VIII). L'évolution de la densité optique à $1510 \mathrm{~cm}^{-1}$ (fig 10b) montre que la vitesse de coupure des noyaux aromatiques est fonction de la quantité d'oxygène présent dans la cellule. Ainsi, la dégradation est plus marquée dans l'oxygène que dans l'air et est très faible dans l'azote (tableau VIII).

Durant les 3 premières $\mathrm{h}$ d'irradiation, la nature de l'atmosphère présente dans la cellule n'intervient pas dans la variation de l'absorption à $1660 \mathrm{~cm}^{-1}$ (fig 10c). Pour une irradiation plus longue, cette variation de la densité optique à $1660 \mathrm{~cm}^{-1}$ ne semble plus proportionnelle à la concentration en oxygène dans la cellule, l'absorption en présence d'azote se plaçant entre les absorptions des échantillons irradiés sous oxygène et à l'air. La présence d'eau dans l'échantillon irradié dans l'air qui n'a pas subi le pompage sous vide pourrait être responsable de cette anomalie, les vibrations de déformation $\mathrm{H}-\mathrm{O}-\mathrm{H}$ se manifestant à $1638 \mathrm{~cm}^{-1}$.

Pour des temps d'irradiation plus importants, les évolutions de l'absorption à cette 

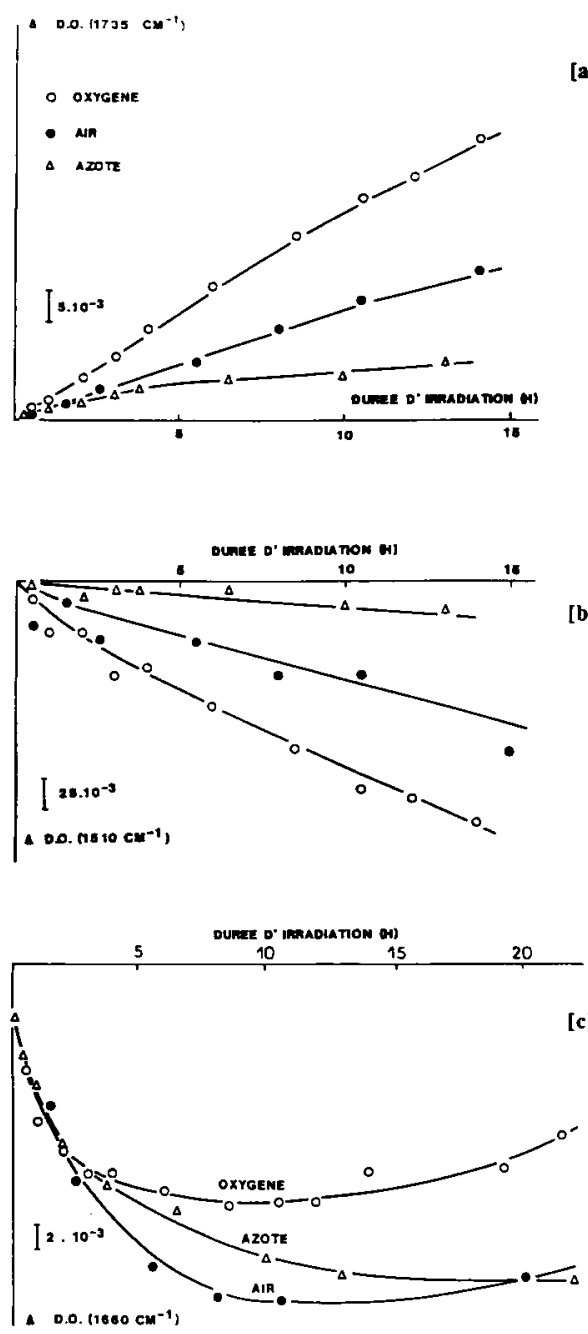

Fig 8. Évolution de la densité optique à 1735 $\mathrm{cm}^{-1}(\mathrm{a})$, à $1510 \mathrm{~cm}^{-1}$ (b) et à $1660 \mathrm{~cm}^{-1}$ (c) en fonction du temps d'irradiation $(O)$ sous oxygène; $(\bullet)$ sous air; $(\Delta)$ sous azote.

longueur d'onde sont différentes entre les échantillons irradiés en présence d'oxygène et d'azote : la densité optique de l'échantillon décroît de façon continue sous azote alors qu'en atmosphère d'oxy-
Tableau VIII. Vitesse d'évolution de la densité optique à $1735 \mathrm{~cm}^{-1}$ et $1510 \mathrm{~cm}^{-1}$ avec le temps d'irradiation ( $\triangle \mathrm{DO}$ par heure d'irradiation avec un flux moyen de $10 \mathrm{~mW} / \mathrm{cm}^{2}$ à $360 \mathrm{~nm}$ ).

\begin{tabular}{lll}
\hline & $v 1735 \mathrm{~cm}^{-1}$ & $v 1510 \mathrm{~cm}^{-1}$ \\
\hline Oxygène & $3,6.10^{-3}$ & $-1,54 \cdot 10^{-3}$ \\
Air & $1,8 \cdot 10^{-3}$ & $-0,83.10^{-3}$ \\
Azote & $1,25.10^{-3}$ & $-0,21.10^{-3}$ \\
\hline
\end{tabular}

gène ou d'air, la densité optique passe par un minimum pour une durée d'irradiation d'environ $10 \mathrm{~h}$. L'augmentation de la densité optique est liée à la présence d'oxygène, en effet, l'introduction d'air dans la cellule lors de l'irradiation sous azote provoque tout d'abord une diminution rapide de l'absorption, suivie d'une augmentation comparable à celle observée lors de l'irradiation sous atmosphère d'oxygène ou d'air (fig 9).

Une autre approche de l'influence de l'oxygène peut être faite en comparant les variations des absorptions à $1735 \mathrm{~cm}^{-1}$ et à $1510 \mathrm{~cm}^{-1}$ d'échantillons irradiés en cellule fermée contenant soit de l'air (concentration en oxygène limitée) soit de l'oxygène pur et en cellule ouverte (tableau IX).

Dès les premières $\mathrm{h}$ d'exposition, l'augmentation de la densité optique à 1735 $\mathrm{cm}^{-1}$ est 2 fois plus importante lorsque l'irradiation a lieu dans l'oxygène ou en cellule ouverte qu'en présence d'un volume d'air limité. La diminution de la bande à 1510 $\mathrm{cm}^{-1}$ est environ 4 fois plus importante quand l'irradiation a lieu en cellule ouverture ce qui confirme la nécessité d'un fort apport d'oxygène dans les mécanismes de dégradation des structures aromatiques.

La séparation des domaines visible et ultraviolet du rayonnement émis par la lampe réalisée à l'aide de filtres montre que l'irradiation à la lumière visible (fig 10a) a peu d'effet sur les modifications 


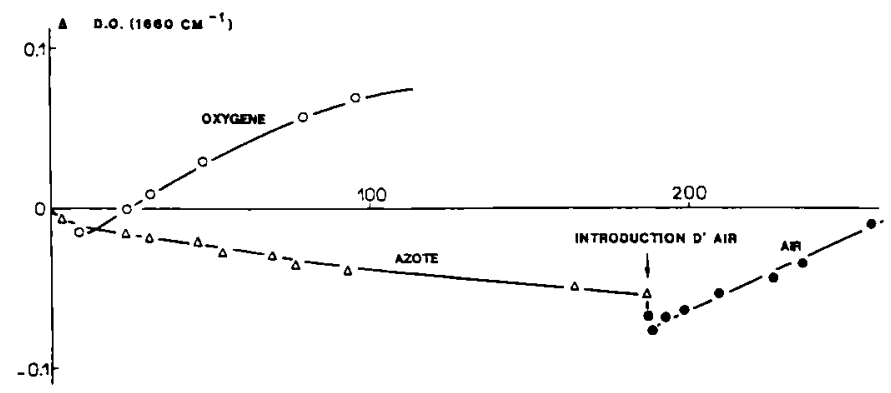

Fig 9. Influence de l'oxygène sur les variations de la densité optique à $1660 \mathrm{~cm}^{-1}$ en fonction du temps d'irradiation.

Tableau IX. Influence de la concentration en oxygène sur les variations d'absorption à $1735 \mathrm{~cm}^{-1}$ et $1510 \mathrm{~cm}^{-1} \mathrm{~d}^{\prime} \mathrm{un}$ échantillon de bois d'Abies grandis irradié.

\begin{tabular}{|c|c|c|c|c|c|c|}
\hline \multirow{4}{*}{$\begin{array}{l}\quad \text { Durée } \\
\text { d'irradiation } \\
\text { en heures }\end{array}$} & \multicolumn{6}{|c|}{$\Delta D O=(D O$ échantillon irradié $-D O$ échantillon avant irradiation $) \times 10^{3}$} \\
\hline & \multicolumn{3}{|c|}{$1735 \mathrm{~cm}^{-1}$} & \multicolumn{3}{|c|}{$1510 \mathrm{~cm}^{-1}$} \\
\hline & \multicolumn{2}{|c|}{ Cellule fermée } & \multirow{2}{*}{$\begin{array}{l}\text { Cellule } \\
\text { ouverte }\end{array}$} & \multicolumn{2}{|c|}{ Cellule fermée } & \multirow{2}{*}{$\begin{array}{l}\text { Cellule } \\
\text { ouverte }\end{array}$} \\
\hline & $\mathrm{O}_{2}$ & Air & & $\mathrm{O}_{2}$ & Air & \\
\hline 0,5 & 2,0 & 1,20 & 2,3 & $-1,8$ & $-1,0$ & $-4,0$ \\
\hline 1 & 3,0 & 1,75 & 4,2 & $-2,8$ & $-1,5$ & $-5,0$ \\
\hline 5 & 16,5 & 8,50 & 18,0 & $-8,5$ & $-4,2$ & $-15,0$ \\
\hline 10 & 32,5 & 17,00 & 34,0 & $-14,5$ & $-7,5$ & $-26,5$ \\
\hline 20 & 60,5 & 30,00 & 59,0 & $-24,7$ & $-14,2$ & $-44,5$ \\
\hline
\end{tabular}

de la structure chimique de l'échantillon. La faible augmentation de l'absorption à $1735 \mathrm{~cm}^{-1}$ suggère la création de groupements carbonyles. Des composés de type cétone aromatique $\left(1660 \mathrm{~cm}^{-1}\right)$ semblent également produits en petite quantité. Les évolutions du spectre d'absorption infrarouge sous une irradiation en lumière ultraviolette (fig 10b) sont beaucoup plus marquées. Notamment, nous pouvons remarquer la forte absorption dans les ré- gions $1735 \mathrm{~cm}^{-1}$ et $1650 \mathrm{~cm}^{-1}$ ainsi qu'une diminution à $1510 \mathrm{~cm}^{-1}$.

\section{Suivi de la photodégradation du bois d'Abies grandis par spectroscopie d'absorption ultraviolet-visible}

Les spectres d'absorption ultraviolet-visible d'une coupe de bois d'Abies grandis non irradiée (fig 11) présentent une structure 
[a]

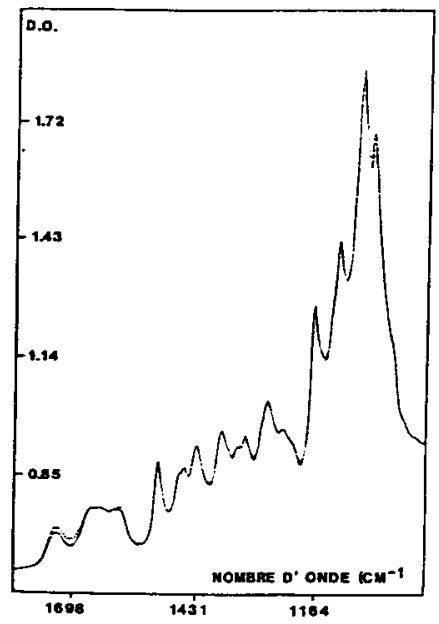

[b]

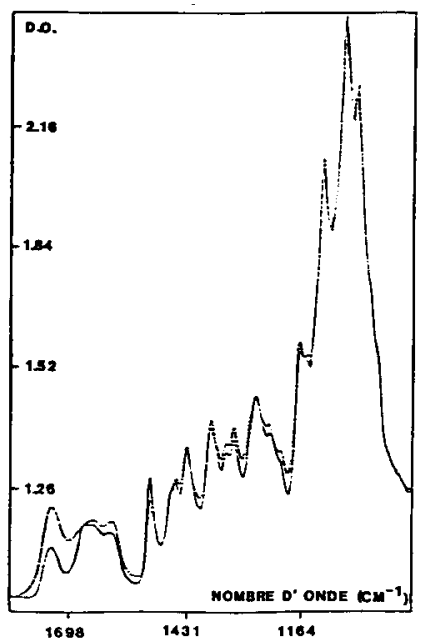

Fig 10. Influence de la nature du rayonnement: spectres d'absorption infrarouge d'une coupe de bois d'Abies grandis - avant irradiation; --après $315 \mathrm{~h}$ d'irradiation en lumière visible (a) et en lumière ultraviolette (b).

continue avec des épaulements peu marquées et des maxima mal séparés. Dans la partie visible du spectre $(400-700 \mathrm{~nm})$, l'absorption est très faible. Après une irradiation continue de $2000 \mathrm{~h}$ avec un flux lu-

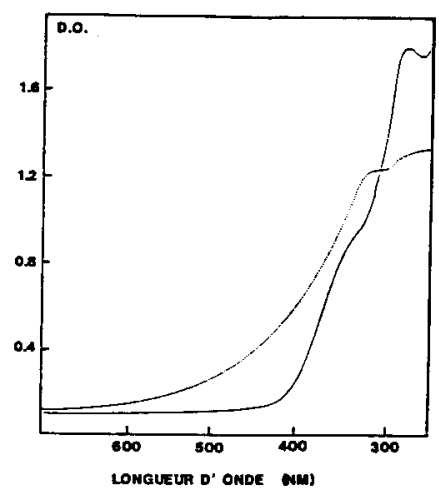

Fig 11. Spectre d'absorption UV-visible d'une coupe de bois d'Abies grandis - avant irradiation; --- après $2000 \mathrm{~h}$.

mineux moyen de $3,5 \mathrm{~mW} / \mathrm{cm}^{2}$ (fig 11), nous notons une augmentation de l'absorption dans tout le domaine visible qui témoigne d'une modification de couleur de l'échantillon. Dans le domaine ultraviolet, l'absorption croît jusqu'à $320 \mathrm{~nm}$ et chute de 300 à $250 \mathrm{~nm}$. Pour mieux localiser les longueurs d'onde où évolue l'absorption, nous pouvons construire les courbes de différence (spectre de l'échantillon irradié spectre du même échantillon avant irradiation) (fig 12). Ainsi, après $2000 \mathrm{~h}$ d'irradiation, la densité optique à $435 \mathrm{~nm}$ a augmenté de $174 \%$ par rapport à sa valeur initiale alors que celle à $280 \mathrm{~nm}$ a chuté de $28 \%$. Cette longue irradiation donne la tendance finale de la modification de couleur du bois d'Abies grandis exposé à la lumière solaire. L'évolution de ces courbes de différence d'absorption avec le temps d'irradiation (fig 13) permet de suivre la chronologie des différentes étapes qui interviennent dans la modification des spectres d'absorption ultraviolet-visible. Nous notons également un déplacement régulier de la position des extrêmes durant l'irradiation (tableau X). De la même façon, 


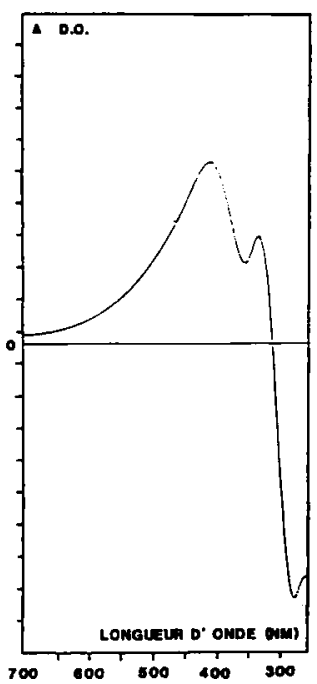

Fig 12. Courbe de différence du spectre d'absorption UV-visible d'une coupe de bois d'Abies grandis irradiée $2000 \mathrm{~h}$ et du spectre d'absorption UV-visible de la même coupe non irradiée.

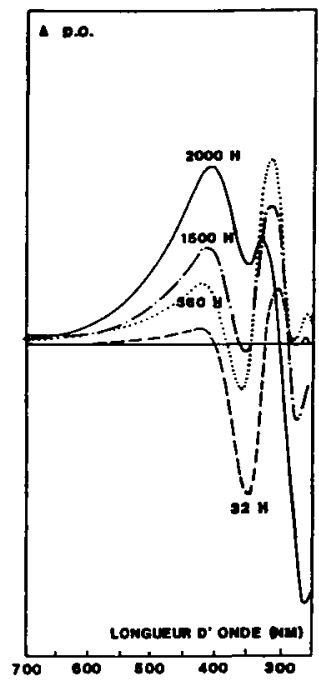

Fig 13. Évolution des courbes de différence (spectre d'absorption UV-visible de l'échantillon irradié - spectre d'absorption UV-visible de l'échantillon non irradié) avec le temps d'irradiation.
Tableau X. Évolution de la longueur d'onde des extrema du spectre d'absorption UV-visible du bois d'Abies grandis en fonction du temps d'irradiation (flux lumineux moyen de $10 \mathrm{~mW} / \mathrm{cm}^{2}$ à $360 \mathrm{~nm}$ ).

\begin{tabular}{rllll}
\hline $\begin{array}{c}\text { Temps } \\
\text { d'irradiation } \\
\text { en } h\end{array}$ & \multicolumn{4}{c}{$\begin{array}{c}\text { Position des extrema } \\
\text { (en mn) }\end{array}$} \\
\hline & & & & \\
4 & 435 & 650 & 305 & \\
32 & 425 & 350 & 305 & 265 \\
360 & 415 & 355 & 310 & 260 \\
1500 & 410 & 355 & 315 & 260 \\
2000 & 405 & 350 & 325 & 260 \\
\hline
\end{tabular}

nous avons suivi la photodégradation de la lignine et de la cellulose par spectroscopie d'absorption ultraviolet-visible. Afin de mieux visualiser les changements intervenus lors de l'irradiation, nous avons tracé, en fonction du temps d'irradiation, les évolutions de la courbe de différence lignine irradiée - lignine avant irradiation (fig 14a) : le minimum à $525 \mathrm{~nm}$ s'accentue avec le temps d'irradiation tandis que les absorptions à $350 \mathrm{~nm}$ et $280 \mathrm{~nm}$ chutent de façon remarquable. Comme pour les spectres du bois d'Abies grandis, la position de certains extrêmes varie avec le temps d'irradiation (tableau XI). Par contre, le spectre d'absorption ultraviolet-visible de la cellulose est très peu marqué par une irradiation de type solaire (fig 14b). On observe un minimum à $290 \mathrm{~nm}$ qui pourrait traduire une dégradation de la liaison acétale avec formation de photoproduits carbonylés absorbant vers $305 \mathrm{~nm}$. L'absorption dans la zone spectrale visible est due en grande partie à des phénomènes de diffusion de la lumière, les échantillons étant réalisés par pastillage d'un mélange de cellulose en poudre et de bromure de potassium. En effet, sous forme d'un film de cellophane (cellulose pure), la cellulose n'absorbe pas la lumière visible. 
[a]

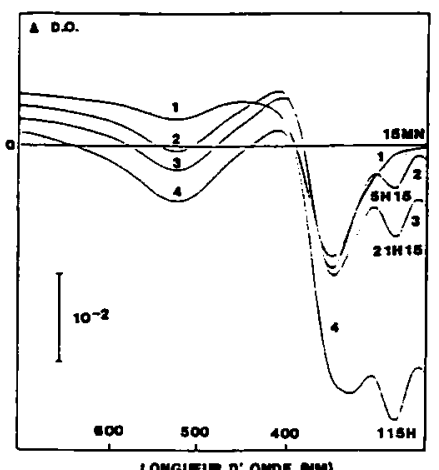

[b]

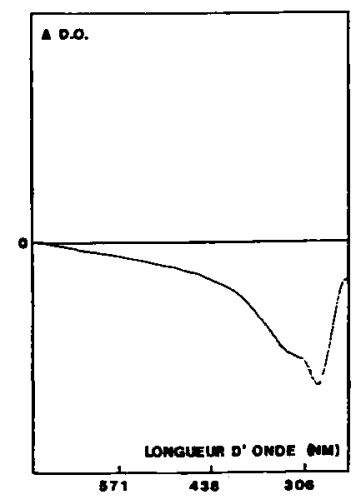

Fig 14. Ėvolution de la courbe de différence du spectre d'absorption UV-visible (lignine irradiée - lignine non irradiée) (a) et (cellulose irradiée cellulose non irradiée) (b) en fonction du temps d'irradiation.

Comme nous l'avons déjà noté en spectroscopie d'absorption infrarouge, des 2 constituants principaux, la lignine est celui dont l'évolution spectrale sous irradiation solaire est la plus proche de celle du bois (fig 15): hormis la bande de décoloration à $525 \mathrm{~nm}$ observée uniquement sur la lignine isolée, les extrêmes se retrouvent aux mêmes longueurs d'onde mais les intensités relatives et les vitesses d'évolution de chaque bande sont différentes.
Tableau XI. Évolution de la longueur d'onde des extrema du spectre d'absorption UV-visible des lignines du bois d'Abies grandis en fonction du temps d'irradiation (flux lumineux moyen de $10 \mathrm{~mW} / \mathrm{cm}^{2}$ à $360 \mathrm{~nm}$ ).

\begin{tabular}{lll} 
Durée d'irradiation $(h)$ & 0,25 & 115 \\
\hline & & \\
\hline & & \\
Position des extrêmas (mm) & 525 & 525 \\
& 450 & 410 \\
& 350 & 333 \\
& 280 & 280
\end{tabular}

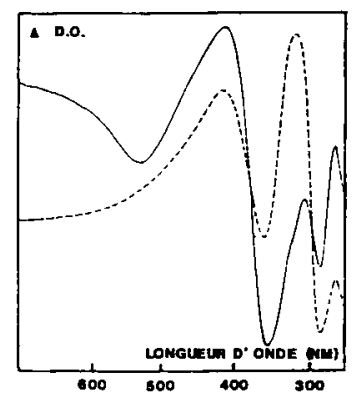

Fig 15. Courbe de différence de spectres d'absorption UV-visible --- lignine irradiée - lignine non irradièe; - Abies grandis irradié - Abies grandis non irradié.

La cellulose n'intervient pas de façon notable dans la photochimie du bois. Pourtant, à l'état isolé, la cellulose subit une photodégradation au rayonnement solaire qui a pu être suivi par la perte de masse (Hon, 1979) ou la diminution du degré de polymérisation (Desai et Shields, 1969). Dans le bois, la photochimie de la cellulose est totalement modifiée par la présence de lignine. II a pu être montré (Hon, 1975) que les molécules de lignine sont capables de désactiver l'état excité de 
la cellulose par un phénomène de transfert d'énergie.

rayonnement lumineux

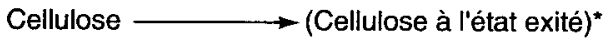
absorbé par la cellulose lignine
(transfert d'énergie) Cellulose + (lignine à l'état excité) ${ }^{\star}$

produits finaux

À ces longueurs d'onde d'irradiation, l'excitation directe de la lignine est aussi possible :

rayonnement lumineux

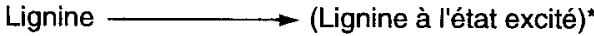
absorbé

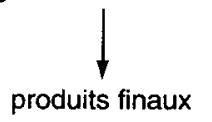

Sous l'action du rayonnement solaire, les photoproduits formés dans le bois d'Abies grandis auraient donc pour origine exclusivement l'état excité des molécules de lignine. La lignine serait donc utilisée par la nature pour "protéger» du rayonnement solaire la cellulose, molécule par laquelle le glucose est mis en réserve.

Ces transferts d'énergie permettent aussi de penser que la photodégradation ne se limite pas uniquement aux couches de bois atteintes directement par le rayonnement. L'excitation électronique initialement localisée sur une molécule de lignine en un point $A$ peut se retrouver en un point $B$ sur une autre molécule de lignine. Dans un milieu condensé comme le bois, ce sont ces transferts d'énergie d'une molécule de lignine à une autre répétés $x$ fois qui permettent de faire migrer l'énergie d'un point $A$ à un point $B$ sans déplacement de la molécule (Lablache-Combier, 1985).
Pour essayer de corréler les résultats obtenus en spectroscopie infrarouge, nous avons suivi l'influence de l'oxygène sur l'évolution du spectre d'absorption ultraviolet-visible d'une coupe de bois d'Abies grandis dans les mêmes conditions opératoires (fig 16). Ces courbes de différence montrent que la présence de l'oxygène dans la cellule d'irradiation intervient dans l'évolution des mécanismes de photocoloration:

- le maximum d'absorption dans le visible au voisinage de $430 \mathrm{~nm}$ est 4 fois plus important après $33 \mathrm{~h}$ d'irradiation dans l'oxygène pur que dans l'azote; d'ailleurs, visuellement l'échantillon irradié en présence de gaz inerte n'a pas jauni contrairement aux 2 autres cas. Ce maximum se déplace avec la concentration en oxygène; après $33 \mathrm{~h}$ d'irradiation, la longueur d'onde de ce maximum est de $430 \mathrm{~nm}$ dans l'azote, $425 \mathrm{~nm}$ dans l'air et $410 \mathrm{~nm}$ dans l'oxygène pur;

- dans le domaine ultraviolet, on observe :

* une décroissance de l'absorption à $350 \mathrm{~nm}$ dans les 3 atmosphères étudiées;

* une augmentation de l'absorption à $305 \mathrm{~nm}$ d'autant plus marquée que la

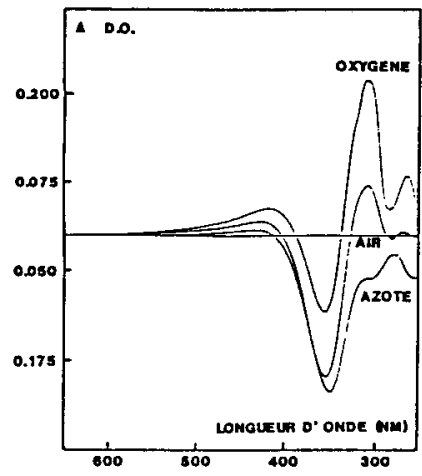

Fig 16. Courbe de différence des spectres d'absorption UV-visible (Abies grandis irradié $33 \mathrm{~h}-$ Abies grandis non irradié) en fonction de la nature de l'atmosphère d'irradiation. 
concentration en oxygène dans la cellule est importante,

" une diminution de l'absorption à $280 \mathrm{~nm}$ corrélée à la concentration en oxygène,

* une augmentation de la densité optique à $265 \mathrm{~nm}$ dans le cas de l'irradiation sous oxygène et dans l'air. En revanche, en présence d'azote, l'absorption à $255 \mathrm{~nm}$ diminue.

L'étude de l'évolution de l'absorption dans le visible à $430 \mathrm{~nm}$ (fig 17a) montre qu'en atmosphère d'azote, un état stationnaire est atteint après $16 \mathrm{~h}$ d'irradiation, ce n'est pas le cas en présence.d'oxygène ou d'air même après $30 \mathrm{~h}$ d'exposition au rayonnement. En début d'irradiation, la vitesse d'accroissement de la densité optique à cette longueur d'onde paraît indépendante de la nature de l'atmosphère gazeuse (fig 17b). De plus, l'allure générale de la courbe de différence obtenue en début d'irradiation sous azote est identique à celle obtenue dans l'air, ce qui indique que les premières étapes de la photodégradation du bois d'Abies grandis ne sont pas affectées par la présence d'oxygène.
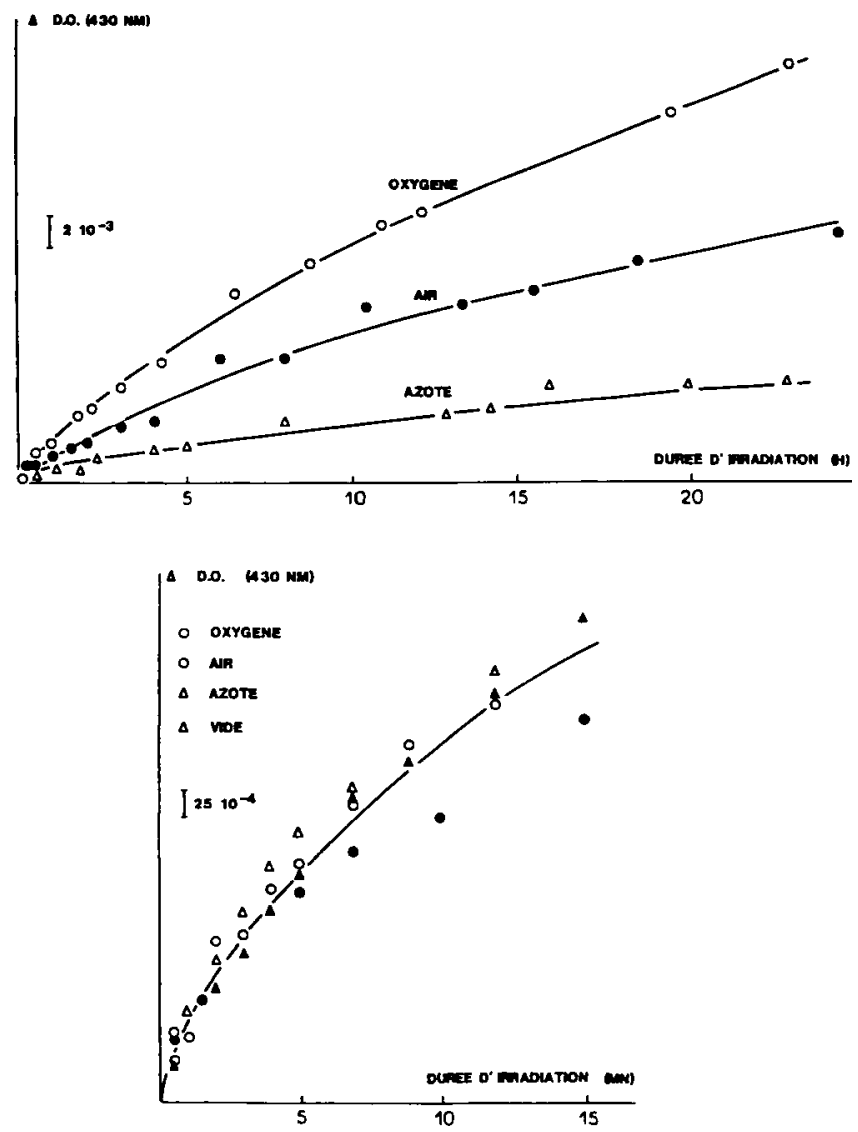

Fig 17. Évolution de l'absorption à $430 \mathrm{~nm}$ sous différentes atmosphères en fonction du temps d'irradiation. 
Les spectres d'absorption ultravioletvisible du bois d'Abies grandis sont également sensibles à la nature spectrale du rayonnement d'excitation. L'absorption dans le domaine visible n'est pas affectée par l'irradiation à des longueurs d'onde > $400 \mathrm{~nm}$. En revanche, cette irradiation dans le domaine visible génère des modifications de l'absorption dans l'ultraviolet : décroissance à $340 \mathrm{~nm}$ et augmentation à $305 \mathrm{~nm}$.

Si ces mêmes modifications sont plus marquées en exposant l'échantillon uniquement au rayonnement ultraviolet émis par la source lumineuse, nous observons en plus 2 bandes d'absorption centrées à $410 \mathrm{~nm}$ et $265 \mathrm{~nm}$. Ceci indique que les changements de coloration du bois d'Abies grandis doivent être imputés au domaine ultraviolet du rayonnement solaire. L'irradiation à la lumière visible $(62 \%$ du flux énergétique total émis par la lampe) ne génère pas de composés colorés puisqu'il n'y a pas de modification de l'absorption dans le domaine visible. Visuellement, seul l'échantillon irradié par le rayonnement ultraviolet présente un jaunissement que l'on a pu quantifier dans le système CIE LAB (tableau XII). Bien qu'en plus faible quantité, les photons ultraviolets sont les plus actifs dans les changements de coloration du bois.

Tableau XII. Influence de la nature spectrale du rayonnement sur les différences des coordonnées chromatiques entre un échantillon irradié et non irradié de bois d'Abies grandis.

\begin{tabular}{ccc}
\hline & $\begin{array}{c}\text { Lumière } \\
\text { visible }\end{array}$ & $\begin{array}{c}\text { Lumière } \\
\text { ultraviolette }\end{array}$ \\
\hline$\Delta \mathrm{L}$ & $-1,1$ & $-3,3$ \\
$\Delta \mathrm{a}$ & 0,26 & 0,63 \\
$\Delta \mathrm{b}$ & 0,37 & 4,19 \\
$\Delta \mathrm{E}$ & 1,2 & 5,4 \\
Appréciation visuelle* & légère & appréciable \\
\hline
\end{tabular}

\section{DISCUSSION}

L'ensemble des résultats expérimentaux obtenus montre que les modifications de couleur du bois d'Abies grandis soumis à une irradiation de type solaire doivent être principalement attribuées à la photodégradation de la lignine. Des études photochimiques réalisées sur d'autres espèces résineuses (Leary, 1967, 1968a, b; Hon et $a l, 1984$ ) avaient déjà dégagé l'importance de l'influence de ce constituant du bois. En s'appuyant sur les résultats obtenus sur le comportement photochimique de la lignine soit directement (Kringstadt et Lin, 1970; Lin et Kringstadt, 1970; Gellersted et Petterson, 1977, 1980; Neumann et al, 1986a,b; Castellan et al, 1990) soit à l'aide de molécules modèles (Lin et Krinsgtadt, 1970, 1971; Gellerstedt et Petterson, 1975; Castellan et al, 1985; Vanucci et al, 1988; Castellan et al, 1989a,b), nous pouvons postuler que les photoproduits colorés formés lors de l'irradiation du bois d'Abies grandis ont pour origine exclusivement un radical stable de type phénoxy- : le radical gaïacoxy- (fig 19). Nous avons pu confirmer par spectroscopie de résonance paramagnétique électronique l'aspect radicalaire de la photodégradation du bois d'Abies grandis. Le signal observé sur l'échantillon de bois a la même allure que celui obtenu lors de l'irradiation de la lignine (fig 18) tandis qu'aucun signal n'est détecté lors de l'irradiation de la cellulose dans les même conditions opératoires. Les

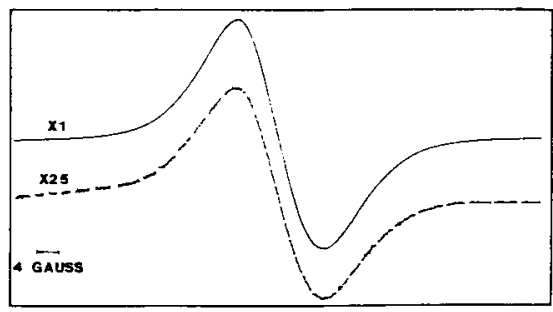

Fig 18. Singal RPE après 15 min d'irradiation lignine; --- Abies grandis. 
irradiations sous oxygène ou sous azote conduisent à des spectres de résonance paramagnétique électronique de forme identique ce qui indique la formation des mêmes espèces radicalaires. Toutefois, la concentration en radicaux est plus importante dans l'oxygène.

En présence d'oxygène, les radicaux gaïacoxy- se décomposent en conduisant à des photoproduits quinoniques colorés (Leary, 1968b; Gellersted et Petterson, 1977, 1980) qui absorbent à des Iongueurs d'onde de l'ordre de $410 \mathrm{~nm}$ (fig 19). Ainsi, l'absorption à $430 \mathrm{~nm}$ est composée des absorptions dues au radical gaiiacoxy- $(\lambda \max \approx 430 \mathrm{~nm})$ et aux photoproduits colorés. Nous avons effectivement observé que le maximum d'absorption à $430 \mathrm{~nm}$ en atmosphère d'azote se
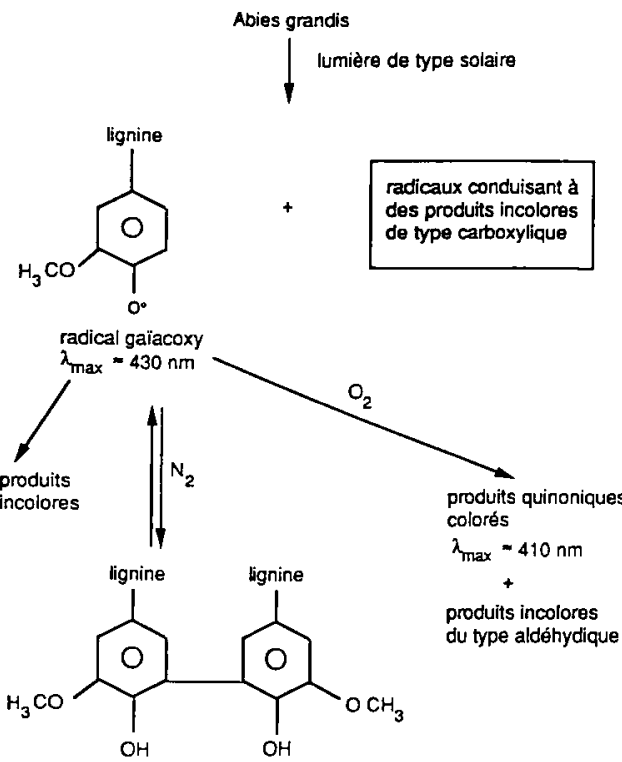

diphényl incolore

Fig 19. La désactivation du radical gaïacoxy- : modèle mécanistique du comportement photochimique du bois $d^{\prime *}$. déplaçait avec le temps d'irradiation vers les courtes longueurs d'onde en présence d'oxygène. Ce déplacement est d'autant plus important que la concentration en oxygène est plus élevée.

En absence d'oxygène, le seul composé coloré est le radical gaïacoxy-. Sa désactivation conduit à des produits incolores, en particulier un composé diphényle substitué formé par la réaction équilibrée de dimérisation mise en évidence lors de l'irradiation de molécules modèles de la lignine (Bouas-Laurent et Castellan, 1987) (fig 19).

Les modifications de couleur intervenues lors d'un stockage à l'obscurité après irradiation confirment l'existence de cet équilibre dans le bois d'Abies grandis. Après irradiation sous atmosphère d'azote, l'absorption à $430 \mathrm{~nm}$ augmente sans déplacement durant la stockage. Une nouvelle irradiation de cet échantillon provoque la diminution régulière de la densité optique à $430 \mathrm{~nm}$. Lors du stockage, un état d'équilibre s'établit entre la forme dimère et la forme radicalaire. Lorsque nous soumettons l'échantillon à une nouvelle irradiation, nous créons une perturbation qui déplace cet équilibre.

Durant un stockage à l'obscurité sous oxygène après irradiation dans ce même gaz, l'intensité de l'absorption à $430 \mathrm{~nm}$ augmente mais nous notons un déplacement du maximum d'absorption vers les faibles longueurs d'onde $\left(\lambda_{\max } \longrightarrow 410\right.$ $\mathrm{nm})$. Cette augmentation de la densité optique est plus importante dans l'oxygène que dans l'air. Si nous soumettons de nouveau au rayonnement lumieux l'échantillon stocké, la diminution de l'absorption mesurée à $430 \mathrm{~nm}$ est beaucoup plus lente que dans le cas de l'atmosphère d'azote. Durant le stockage, les radicaux gaïacoxy- se combinent à l'oxygène pour donner les dérivés quinoniques qui absorbent plus fortement et à une longueur d'onde plus faible. 
La formation de ces dérivés est aussi responsable de l'augmentation de l'absorption à $260 \mathrm{~nm}$, observée uniquement lors de l'irradiation en présence d'oxygène (fig 16). De même, la diminution de la densité optique à $280 \mathrm{~nm}$ corrélée à la concentration en oxygène est attribuée à la désaromatisation lors de la formation de ces dérivés quinoniques.

Les résultats obtenus en spectroscopie d'absorption infrarouge confirment ces mécanismes : l'augmentation de l'absorption à $1660 \mathrm{~cm}^{-1}$ observée uniquement sous atmosphère d'oxygène correspond à la formation des dérivés quinoniques (vibration d'élongation de la liaison $\mathrm{C}=\mathrm{O}$ dans les quinones : $1660-1680 \mathrm{~cm}^{-1}$ ) (Silverstein et al, 1981). De même, la diminution de la densité optique à $1510 \mathrm{~cm}^{-1}$ est liée à la désaromatisation de la lignine d'autant plus marquée que la concentration en oxygène est élevée.

La formation des composés carbonylés non colorés de type carboxylique et aldéhydique induit l'augmentation de l'absorption à $1735 \mathrm{~cm}^{-1}$ en spectroscopie infrarouge et à $305 \mathrm{~cm}^{-1}$ en spectroscopie ultraviolette. Cette augmentation est aussi liée à la concentration en oxygène lors de l'irradiation.

\section{CONCLUSION}

En choisissant le bois d'Abies grandis comme modèle du comportement photochimique du bois pour s'affranchir de l'influence des substances extractibles colorées, nous avons pu montrer que les lignines sont responsables des modifications de couleur du bois exposé au rayonnement de type solaire. Par contre, la cellulose ne semble pas intervenir dans la détérioration de l'aspect coloré du bois.

Nous avons pu proposer un mécanisme en accord avec les résultats obtenus sur des molécules modèles des lignines. Cette étude a permis de faire une revue complète des différentes réactions photochimiques intervenant dans la photodégradation du bois et nous a conduits à une proposition sur 1 des rôles des lignines peu évoqué jusqu'à présent : le rôle protecteur de la cellulose vis-à-vis de l'irradiation de type solaire.

Les mécanismes proposés montrent également que nous pouvons avoir apparition d'une couleur au sein du bois sans avoir un abaissement significatif du degré de polymérisation de la macromolécule ligneuse : la coloration peut provenir d'un réarrangement de la structure phénolique de la molécule de lignine en structure quinonique responsable de l'absorption de la lumière visible. Ainsi, les modifications de couleur ne sont pas directement liées à l'altération des propriétés mécaniques du bois.

$\mathrm{Ce}$ jaunissement lui-même peut être considéré comme un moyen de protection du bois vis-à-vis de la lumière. Par effet de filtre interne dû à cette coloration, la pénétration des photons est affaiblie et la photodégradation sera limitée à la surface de l'échantillon.

\section{REMERCIEMENTS}

Les auteurs remercient le laboratoire de biochimie de I'ENSAIA de Nancy et le centre de recherches sur les macromolécules végétales (CERMAV) de Grenoble qui ont fourni respectivement la cellulose Solva Bloc BW200 extraite de bois de peuplier (Populus canescens $\mathrm{Sm}$ ) et les hémicelluloses provenant de bois de séquoïa (Sequoïa sempervirens) et de roseau (Arundi donax).

\section{RÉFÉRENCES}

Bouas-Laurent H, Castellan A (1987) Photoyellowing of Paper. Séminaire interlaboratoire INRA-Centre de Grignon 
Castellan A, Vanucci, Desvergne JP, BouasLaurent $H$, Hauteville $M$, Chadenson $M$ (1985) Étude photochimique de dimères modèles de lignine de type $\alpha-0-4$ et $\beta-0-4 \quad 0$ méthylés. C R Acad Sci 301 (II), 21-26

Castellan A, Colombo N, Cucuphat C, Forniet de Violet $P$ (1989a) Photodegradation of lignin: a photochemical study of phenolic $\alpha$ carbony $\beta-0-4$ lignin model dimer 4-hydroxy3-methoxy-a-(2' methoxy phenoxy) acetophenone. Holzforschung 43, 179-185

Castellan A, Colombo N, Forniet de Violet $P$, Nourmamode A, Bouas-Laurent H (1989b) Photodegradation of lignin: a photochemical study of bleached CTMP and lignin model molecules. Tappi Proc, Wood Pulping Chem $1,421-430$

Castellan A, Nourmamode A, Forniet de Violet $P$ (1990) Photodégradation en matrice solide carbohydrate de lignine extraite du pin maritime d'Aquitaine; aspect moléculaire. Acte du $3^{e}$ Coll Sci Industrie Bois, Bordeaux 1,331

Chow SZ (1971) Infrared spectra characteristics and surface inactivation of wood at high temperatures. Wood Sci Technol 5, 27-39

Christment A (1988) Science et technologie de la mesure de la couleur. Bulletin Sci Coul $\mathrm{n}^{\circ}$ 1, 9-12

Decarreau JF (1988) Le système CIE LAB. Bull Sci Coul 1, 13-15

Desai RL, Shields JA (1969) Photochemical degradation of cellulose material. Makromol Chem 122, 134-144

Dirckx O (1984) Mise en évidence de la photodégradation et des variations de couleur du bois sous irradiation UV par des techniques spectroscopiques. DEA Sci Bois - Université de Nancy 1

Dirckx O (1988) Étude du comportement photochimique de l'Abies grandis sous irradiation solaire. Thèse de $3^{\mathbf{e}}$ Cycle en Sci Bois Univ Nancy $I$

Dordet $Y$ (1990) La colorimétrie. Principes et applications. Eyrolles, Paris

Fengel D, Wegener G (1984) Wood Chemistry, Ultrastructure, Reactions. De Gruyter, Berlin

Flot JL (1988) La couleur du chêne de tranchage français. INRA, Centre de Champenoux, Station de recherches sur la qualité du bois, Rapport de stage ENITEF
Gaillard JM (1984) Photodégradation de systèmes bois-finitions (résines alkydes). Thèse de $3^{e}$ cycle-Sci Bois - Univ Nancy 1

Gellerstedt G, Pettersson EL (1975) Light induced oxidation of lignin. The behaviour of structural units contraining a ring conjugated double bond. Acta Chem Scand B29, 10051010

Gellerstedt G, Pettersson EL (1977) Light induced oxidation of lignin. Part 2: the oxidative degradation of aromatic rings. Svenks Papperstid 80, 15-21

Gellerstedt G, Pettersson EL (1980) Autooxidation of lignin. Svenks Papperstid 83, 314-318

Gierer J, Lin SY (1972) Photodegradation of lignin. A contribution to the mechanism of chromophore formation. Svenks Papperstid 75, 233-239

Harrington KJ, Higgins HG, Michell AJ (1964) Holzforschung 18(4), 108-113

Hofmann P (1987) Mesure de la variabilité de la couleur du bois : méthodologie et influences de l'anatomie du plan ligneux et des variations angulaires du débit. DEA Sci bois Univ Nancy I

Hong DNS (1975) Formation of free radicals in photoirradiated cellulose $\mathrm{VI}$ - Effect of lignin. J Polym Sci Polym Chem Ed 13, 2641-2652

Hon DNS (1979) Photooxidative degradation of cellulose: reactions of the cellulosic freeradicals with oxygen. I Polym Sci Polym Chem Ed 17, 441-454

Hon DNS, Chang ST (1984) Surface degradation of wood by ultraviolet light. J Polym Sci Polym Chem Ed 22, 2227-2241

Janin $G$ (1986) La couleur du bois, un facteur clé de qualité. Mesures 61-65

Kovaliski $P(1990)$ Vision et mesure de la couleur, Masson, Paris, $2 e$ ed

Kringstadt KP, Lin SY (1970) Mechanism in the yellowing of high yield pulps by light. Structure and reactivité of free radical intermediates in the photodegradation of lignin. Tappi 53(12), 2296-2301

Lablache-Combier A (1985) Photochimie et polymères. Actual Chim 39-60

Leary GJ (1967) The yellowing of wood by light. Part I. Tappi 50(1), 17-19

Leary GJ (1968a) The yellowing of wood by light. Part II. Tappi 51(6), 257-260 
Leary GJ (1968b) Photochemical production of quinoiid structures in wood. Nature 217, 672673

Le Nest JF, Silvy J, Gandini A (1982) Effets des radiations visibles et proche-ultraviolettes sur les matériaux lignocellulosiques. Actes $d u 1^{\circ}$ colloque Sciences et Industrie du Bois, Grenoble, 367-384

Liang CY, Basste KH, Mc Ginnes EA, Marchessault RH (1968) Tappi, 43(12), 1017-1024

Lin SY, Krinsgstadt KP (1970) Photosensitive groups in lignin and lignin model compounds. Tappi 53(4), 1675-1677

Lin SY, Krinsgstadt KP (1971) Some reactions in the photoinduced discoloration of lignin. Norsk Shogindustri 25(9), 252-256

Marchessault RH (1962) Application of infrared spectroscopy to cellulose and wood polysaccharides. Pure Applic 5, 107-129

Mazet JF (1988) Couleur et qualité des placages de Chêne et étude de leur comportement photochimique. Thèse de Doct Univ Nancy I en Sciences du Bois

Mc Ginnes EA, Rosen HN (1984) Growth quality evaluation of black walnut wood. Part III. An anatomical study of color characteristics of black walnut vener. Wood Fiber Sci 16(1), 48-56

Merlin A, Fouassier JP (1980) Photochemical investigations into cellulosic materials. I. Free radical generation in cellulose by photosensitized excitation. Angew Makromol Chem 86, 109-121

Michell AJ, Ingle HD, Steward CM (1969) Infrared spectra of some woods of taxomic interest. Wood Sci Technol 3, 93-99

Minemura N, Umehara K (1979) Color improvement of wood (report 1): photoinduced disco- loration and its control. $J$ Hokkaido For Prod Res Inst 54(3), 92-145

Nagaty A, El Sayed OH, Ibrahim ST, Mansour OY (1982) Chemical and spectral studies on hemicelluloses isolated from bagasse. Holzforschung 36, 29-35

Nemeth K (1982) Examinations of tree colour in the CIE LAB system. Erdeszeri es paipari 2, 125-135

Neumann MG, De Groote RA, Machado AE (1986a) Flash photolysis of lignin. Part I: decrated solutions of dioxane-lignin. Polym Photochem 7, 401-407

Neumann MG, De Groote RA, Machado AE (1986b) Flash photolysis of lignin. Part II: Oxidative photodegradation of dioxane-lignin. Polym Photochem 7, 461-468

Pecina H (1982) Du pouvoir d'information des spectrogrammes IR sur les modifications chimiques de la structure du bois à l'exemple du traitement thermique. Trad $\mathrm{J}$ Bressard d'après : Holztechnologie 23(2), 78-84

Sarkanen KV, Chang HM, Ericsson B (1967) Species variation in lignins. Part I: IR spectra of guaiaacyl and syringyl models. Tappi 50, 572-575

Schmitt $P$ (1984) Étude des effets du rayonnement de la lumière du jour sur la cellulose et les matières ligneuses. Thèse de $3^{e}$ cycle, INP Grenoble

Silverstein RM, Bassler, Morill TC (1981) Spectometric identification or organic compounds. $4^{\mathrm{e}}$ edn

Vanucci $C$, Forniet de Violet $P$, Bouas-Laurent $H$, Castellan A (1988) Photodegradation of lignin: a photophysical and photochemical study of a non-phenolic $\alpha$-carbonyl $\beta-0-4 \mathrm{li}$ gnin model dimer 3-4 dimethoxy- $\alpha$-(2'methoxyphenoxy-acetophenone. $J$ Photochem Photobiol A: Chem 41, 251-265 